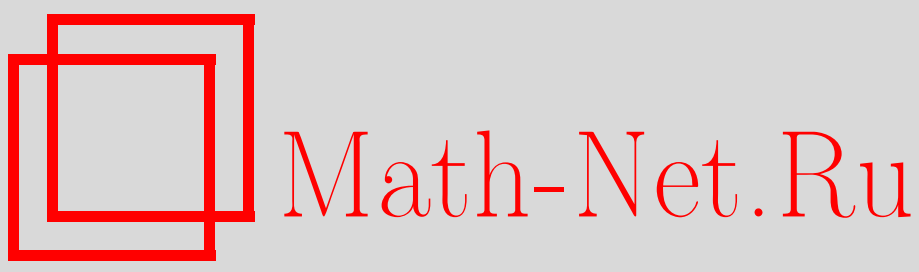

В. И. Скалыга, Аналоги неравенств Марковых и Бернштейна для полиномов в банаховых пространствах, Изв. РАН. Сер. матем., 1997, том 61, выпуск 1, 141-156

DOI: https://doi.org/10.4213/im108

Использование Общероссийского математического портала Math-Net.Ru подразумевает, что вы прочитали и согласны с пользовательским соглашением http: //www. mathnet.ru/rus/agreement

Параметры загрузки:

IP : 3.85 .73 .92

26 апреля 2023 г., 13:00:14 
УДК 517.16

\section{В.И. Скалыга}

\section{Аналоги неравенств Марковых и Бернштейна для полиномов в банаховых пространствах}

Предложены аналоги неравенств А. А. и В. А. Марковых для алгебраических полиномов на кубе в $\mathbb{R}^{m}$ в метриках $l_{2}^{(m)}$ и $l_{1}^{(m)}$, а также для полиномов на выпуклых замкнутых ограниченных телах в банаховом пространстве и на таких же телах с центральной симметрией. Для последних двух случаев получены аналоги неравенства С. Н. Бернштейна.

Библиографияя: 13 наименований.

\section{§1. Введение}

Пусть $\mathfrak{P}_{n}$-множество всех алгебраических полиномов степени не выше $n \in \mathbb{Z}_{+}$, $[a, b] \subset \mathbb{R},\left\|P_{n}\right\|_{[a, b]}=\max _{x \in[a, b]}\left|P_{n}(x)\right|$. Известное (см. [1], [2]) неравенство А. А. и В. А. Марковых утверждает, что для любого $P_{n} \in \mathfrak{P}_{n}$

$$
\left\|P_{n}^{(k)}\right\|_{[a, b]} \leqslant \frac{2^{k} n^{2}\left(n^{2}-1^{2}\right) \ldots\left(n^{2}-(k-1)^{2}\right)}{(b-a)^{k}(2 k-1) ! !}\left\|P_{n}\right\|_{[a, b]},
$$

причем равенство достигается только на полиномах вида

$$
P_{n}(x)=c T_{n}((2 x-a-b) /(b-a))
$$

где $T_{n}(x)=\cos (\operatorname{narccos} x)$.

Неравенство (1) при $k=1$ доказано А. А. Марковым, при $k \geqslant 2$ В. А. Марковым. Для внутренних точек отрезка $[a, b]$ справедливо неравенство С. Н. Бернштейна [3], согласно которому

$$
\left|P_{n}^{\prime}(x)\right| \leqslant n\left\|P_{n}\right\|_{[a, b]} / \sqrt{(x-a)(b-x)} .
$$

Неравенства А. А. Маркова и С. Н. Бернштейна переносились на полиномы многих переменных. O.D. Kellogg [5] показал, что неравенство (1) при $k=1$ переносится на случай шара из гильбертова пространства без изменений. D. R. Wilhelmsen [6] получил следуюший результат: пусть $P_{n}(x)=\sum_{|i| \leqslant n} a_{i} x^{i}$, где $i=$ $\left(i, \ldots, i_{m}\right),|i|=i_{1}+\cdots+i_{m}, x^{i}=x_{1}^{i_{1}} \ldots x_{m}^{i_{m}}$ - полином от $m$ переменных степени не выше $n$, заданный на компактном выпуклом теле $K$ из $\mathbb{R}^{m}$; тогда справедливо неравенство

$$
\sup \left\{\left|D P_{n}(x)\right|: x \in K\right\} \leqslant\left(4 n^{2} / \omega_{K}\right) \sup \left\{\left|P_{n}(x)\right|: x \in K\right\},
$$

где $D$ - производная по любому направлению, а $\omega_{K}$ - ширина $K$. Для случая треугольника этот результат был получен в [7].

Работа выполнена при финансовой поддержке Российского фонда фундаментальных исследований (грант № 93-011-16021). 
Пусть $X$ и $Y$ - банаховы пространства над $\mathbb{R}$. Отображение $U: X^{j} \rightarrow Y$ называется $j$-линейньм $\left(j \in \mathbb{Z}_{+}\right)$, если оно линейно по каждому аргументу при фиксированных остальных и ограничено, т.е. сушествует $M>0$ такое, что справедливо неравенство

$$
\left\|U\left(x_{1}, \ldots, x_{j}\right)\right\|_{Y} \leqslant M\left\|x_{1}\right\|_{X} \ldots\left\|x_{j}\right\|_{X}, \quad x_{k} \in X, \quad k=\overline{1, j} .
$$

Через $\mathfrak{P}_{n}(X, Y)$ обозначим пространство всех полиномов степени не вьше $n$, т.е. множество всех отображений вида

$$
P_{n}(x)=\sum_{j=0}^{n} u_{j}(x, \ldots, x), \quad x \in X
$$

где $u_{j}: X^{j} \rightarrow Y-j$-линейное отображение. В дальнейшем $\left\|P_{n}^{(k)}(x)\right\|_{\mathscr{L}^{k}(X, Y)}-$ норма оператора $k$-й производной по Фреше многочлена $P_{n}$ в точке $x$ в пространстве $\mathscr{L}^{k}(X, Y)$ всех ограниченных $k$-линейных операторов из $X^{k}$ в $Y$. Мы здесь не делаем различия между $\mathscr{L}^{k}(X, Y)$ и изометрически изоморфным ему пространством

$$
\underbrace{\mathscr{L}(X, \mathscr{L}(X \ldots, \mathscr{L}}_{k \text { раз }}(X, Y) \ldots)) .
$$

Если $Y=\mathbb{R}$, то для обозначения производной $k$-го порядка будем применять знак $\nabla^{k}$. Всюду в дальнейшем $K$ - ограниченное, замкнутое, выпуклое тело из $X$, $\partial K$ - его гранища, $B(x, r)$ - замкнутый шар радиуса $r$ с центром в $x$ из $X$. Мы будем использовать следующие характеристики множества $K$ :

$$
\begin{aligned}
r\left(x_{0}\right) & =\sup \left\{r>0: B\left(x_{0}, r\right) \in K\right\}, \\
r(K) & =\sup _{x_{0} \in K} r\left(x_{0}\right), \\
K_{\rho\left(x_{0}\right)} & =\left\{x \in X: x_{0}+\left(x-x_{0}\right) \rho^{-1} \in K\right\}, \quad \text { где } \rho<1, \\
\rho\left(x, x_{0}\right) & =\inf \left\{\rho \geqslant 0: x \in K_{\rho\left(x_{0}\right)}\right\}, \\
b(x) & =\inf _{x_{0} \in K \backslash \partial K}\left[r\left(x_{0}\right)\left(1-\rho\left(x, x_{0}\right)\right)^{1 / 2}\right]^{-1} .
\end{aligned}
$$

Положим

$$
\left\|P_{n}\right\|_{K}=\sup _{x \in K}\left\|P_{n}(x)\right\|_{Y}, \quad\left\|P_{n}^{(k)}\right\|_{K}=\sup _{x \in K}\left\|P_{n}^{(k)}(x)\right\|_{\mathscr{L}^{k}(X, Y)} .
$$

В работе [8] доказаны следующие аналоги неравенств А.А. Маркова и С.Н. Бернштейна:

$$
\begin{aligned}
\left\|P_{n}^{\prime}\right\|_{K} & \leqslant\left(4 n^{2} / r(K)\right)\left\|P_{n}\right\|_{K}, \\
\left\|P_{n}^{\prime}(x)\right\|_{\mathscr{L}(X, Y)} & \leqslant 3 b(x) n\left\|P_{n}\right\|_{K}, \quad x \in K \backslash \partial K .
\end{aligned}
$$

В настоящей работе получены следующие усиления оценок (4) и (5):

$$
\begin{gathered}
\left\|P_{n}^{\prime}\right\|_{K} \leqslant\left(4 n^{2} / \omega_{K}\right)\left\|P_{n}\right\|_{K} \leqslant\left(2 n^{2} / r(K)\right)\left\|P_{n}\right\|_{K}, \\
\left\|P_{n}^{\prime}(x)\right\|_{\mathscr{L}(X, Y)} \leqslant b_{1}(x) n\left\|P_{n}\right\|_{K} \leqslant \sqrt{2} b(x) n\left\|P_{n}\right\|_{K}, \quad x \in K \backslash \partial K,
\end{gathered}
$$


где

$$
\begin{gathered}
\omega_{K}=\inf _{\ell \in X \backslash\{0\}} \sup _{y_{1}, y_{2} \in K, y_{1}-y_{2}=c \ell}\left\|y_{1}-y_{2}\right\|, \\
b_{1}(x)=\inf _{x_{0} \in K \backslash \partial K}\left(1+\rho\left(x, x_{0}\right)\right)^{1 / 2} /\left(r\left(x_{0}\right)\left(1-\rho\left(x, x_{0}\right)\right)^{1 / 2}\right) .
\end{gathered}
$$

Кроме этого в данной работе получены аналоги неравенства (1) для $m$-мерного куба $Q^{m}(a)$ с длиной ребра $a$ в $m$-мерных пространствах $X=\ell_{2}^{(m)}$ и $X=\ell_{1}^{(m)}$. В частности, для $X=\ell_{2}^{(m)}$

$$
\begin{aligned}
\left\|P_{n}^{\prime}\right\|_{Q^{m}(a)} & \leqslant\left(2 n^{2} / a\right)\left\|P_{n}\right\|_{Q^{m}(a)} \\
\left\|P_{n}^{(2)}\right\|_{Q^{m}(a)} & \leqslant\left(4 A / a^{2}\right)\left\|P_{n}\right\|_{Q^{m}(a)},
\end{aligned}
$$

где

$$
A=\min \left(2 n^{2}\left(n^{2}-1\right) / 3, n^{2}(n-1)^{2}\right)
$$

для $X=\ell_{1}^{(m)}$

$$
\begin{aligned}
\left\|P_{n}^{\prime}\right\|_{Q^{m}(a)} & \leqslant\left(2 n^{2} / a\right)\left\|P_{n}\right\|_{Q^{m}(a)}, \\
\left\|P_{n}^{(2)}\right\|_{Q^{m}(a)} & \leqslant\left(4 n^{2}\left(n^{2}-1\right) / 3 a^{2}\right)\left\|P_{n}\right\|_{Q^{m}(a)} .
\end{aligned}
$$

На основе оценок (6) и (7) для центрально-симметричного $K$ из банахова пространства $X$ при $\left\|P_{n}\right\|_{K} \leqslant 1$ получены оценки

$$
\begin{gathered}
\left\|P_{n}^{\prime}\right\|_{K} \leqslant B_{1} / r(K) \leqslant \sqrt{2} n^{2} / r(K), \\
\left\|P_{n}^{\prime}(x)\right\|_{\mathscr{L}(X, Y)} \leqslant \sqrt{2} n /\left(r(K)\left(1-\rho^{2}\left(x, x_{0}\right)\right)^{1 / 2}\right), \quad x \in K \backslash \partial K, \\
\sup _{x \in K}\left\|\left\langle P_{n}^{(2)}(x) h, h\right\rangle\right\|_{Y} \leqslant C_{1} / r^{2}(K) \leqslant C_{2} / r^{2}(K), \quad\|h\|=1,
\end{gathered}
$$

где $x_{0}$ - центр симметрии $K$,

$$
\begin{gathered}
B_{1}=\sup _{\widetilde{P}_{n}}\left|\widetilde{P}_{n t_{1}}^{\prime}(1,1)-\widetilde{P}_{n t_{2}}^{\prime}(1,1)\right|, \\
B_{2}=\sup _{\widetilde{P}_{n}}\left|\left\langle\nabla^{2} \widetilde{P}_{n}(1,1)(1,-1)^{T},(1,-1)^{T}\right\rangle\right|, \\
\widetilde{P}_{n}(t) \in \mathfrak{P}_{n}\left(\mathbb{R}^{2}, \mathbb{R}\right), \quad\left\|\widetilde{P}_{n}\right\|_{Q^{2}} \leqslant 1, \quad Q^{2}=\left\{\left(t_{1}, t_{2}\right) \in \mathbb{R}^{2}:\left|t_{i}\right| \leqslant 1\right\}, \\
C_{0}=n(2 n-1)\left(n^{2}-1\right) / 3, \quad C_{1}=\min \left(2 C_{0}, B_{2}\right), \quad C_{2}=2 \min \left(C_{0}, n^{2}(n-1)^{2}\right) .
\end{gathered}
$$

Показано также, что для параллелограмма в любой норме, заданной на пространстве $\mathbb{R}^{2}$, справедливо неравенство

$$
\left\|P_{n}^{(2)}\right\|_{K} \leqslant\left(C_{2} / r^{2}(K)\right)\left\|P_{n}\right\|_{K}
$$

Чтобы получить перечисленные оценки для любого банахова пространства $Y$, достаточно вывести их для $Y=\mathbb{R}$, что доказывает приведенная ниже лемма 1. 


\section{§2. Вспомогательные леммы}

Лемма 1. Пусть $K \subset X, P_{n} \in \mathfrak{P}_{n}(X, Y),\left\|P_{n}\right\|_{K} \leqslant 1$. Если для любого $\widetilde{P}_{n} \in \mathfrak{P}_{n}(X, \mathbb{R})$ такого, что $\left\|\widetilde{P}_{n}\right\|_{K} \leqslant 1$, справедливь неравенства

$$
\left\|\widetilde{P}_{n}^{(k)}(x)\right\|_{\mathscr{L}^{k}(X, \mathbb{R})} \leqslant C_{k}(x), \quad x \in K, \quad k=\overline{1, n},
$$

то и для $P_{n}$ выполнены неравенства

$$
\left\|P_{n}^{(k)}(x)\right\|_{\mathscr{L}^{k}(X, Y)} \leqslant C_{k}(x), \quad x \in K, \quad k=\overline{1, n} .
$$

ДокАЗАТЕЛЬСТво. Докажем неравенство (10) при $k=1$, для других $k$ доказательство аналогично.

Пусть $y^{*} \in Y^{*}$ и $\left\|y^{*}\right\|_{Y^{*}}=1$. Обозначим $\widetilde{P}_{n}(x)=y^{*}\left(P_{n}(x)\right)$. Тогда

$$
\widetilde{P}_{n}^{\prime}(x) \in \mathscr{L}(X, \mathbb{R}) \quad \text { и }\left\|\widetilde{P}_{n}\right\|_{K} \leqslant 1 .
$$

В силу равенства

$$
\widetilde{P}_{n}^{\prime}(x) h=y^{*}\left(P_{n}^{\prime}(x) h\right), \quad h \in X
$$

(см. $[9$, с. 50]), и (9) имеем для любого нормированного $h \in X$

$$
\left|y^{*}\left(P_{n}^{\prime}(x) h\right)\right| \leqslant C_{1}(x), \quad x \in K
$$

Так как (11) выполняется для любого нормированного $y^{*} \in Y^{*}$, то из него вытекает неравенство

$$
\left\|P_{n}^{\prime}(x) h\right\|_{Y} \leqslant C_{1}(x), \quad x \in K, \quad\|h\|=1 .
$$

Из (12) следует (10).

Введем обозначения. Пусть $x_{1}^{(k-1)}>x_{2}^{(k-1)}>\cdots>x_{n-k+1}^{(k-1)}-$ нули полинома $T_{n}^{(k-1)}(x)-(k-1)$-й производной полинома Чебьшева $T_{n}(x)$;

$$
\begin{aligned}
& d_{n, 1}=n \\
& d_{n, k}=n\left(n^{2}-1^{2}\right) \ldots\left(n^{2}-(k-2)^{2}\right), \quad \text { если } k=2 p+1 \quad(p=1,2, \ldots), \\
& d_{n, k}=n^{2}\left(n^{2}-2^{2}\right) \ldots\left(n^{2}-(k-2)^{2}\right), \quad \text { если } k=2 p \quad(p=1,2, \ldots) .
\end{aligned}
$$

Лемма 2. Пусть $X=\mathbb{R}, \quad P_{n-k}(x) \in \mathfrak{P}_{n-k}(\mathbb{R}, \mathbb{R}) . \quad$ Пусть для $x \in$ $\left[x_{n-k+1}^{(k-1)}, x_{1}^{(k-1)}\right]$ выполнено неравенство

$$
\left|\left(1-x^{2}\right)^{k / 2} P_{n-k}(x)\right| \leqslant 1 .
$$

Тогда справедливы неравенства

$$
\max _{x \in[-1,1]}\left|P_{n-k}(x)\right| \leqslant \max \left(T_{n}^{(k)}(1) / a_{n, k}, b_{n, k}\right)=m_{n, k},
$$


где

$$
\begin{aligned}
& b_{n, k}=\left(1-\left(x_{1}^{(k-1)}\right)^{2}\right)^{-k / 2}, \\
& a_{n, k}=\min _{i=1, n-k+1}\left|T_{n}^{(k)}\left(x_{i}^{(k-1)}\right)\left(1-\left(x_{i}^{(k-1)}\right)^{2}\right)^{k / 2}\right|, \\
& m_{n, k}=T_{n}^{(k)}(1) / a_{n, k} \quad n p u \quad k=\overline{1,6} \quad u \quad k=n-2, n-1, n, k=((2 n-3) / 2)(2(n-1) /(2 n-5))^{(n-2) / 2}, \\
& m_{n, n-2}=((n)-1) / 2 \\
& m_{n, n-1}=(2(n-1))^{n / 2} /(2 n-3)^{(n-1,} \\
& a_{n, k}=c_{n, k} d_{n, k}, \\
& c_{n, 1}=1 ; \quad c_{n, 2}=1 ; \quad c_{n, 3} \geqslant 0.9761 ; \quad c_{n, 4} \geqslant 0.879 ; \\
& c_{n, 5} \geqslant 0.512 ; \quad c_{n, 6} \geqslant 0.482 .
\end{aligned}
$$

ДокАЗАТЕЛЬСТВо. Выпишем для полинома $P_{n-k}(x)$ интерполяционную формулу Лагранжа:

$$
P_{n-k}(x)=\sum_{i=1}^{n-k+1}\left(T_{n}^{(k-1)}(x) P_{n-k}\left(x_{i}\right)\right) /\left(T_{n}^{(k)}\left(x_{i}\right)\left(x-x_{i}\right)\right),
$$

где $x_{i}=x_{i}^{(k-1)}, i=\overline{1, n-k+1}$.

Если $x \in[1,-1] \backslash\left[x_{n-k+1}, x_{1}\right]$, то из (17) и (13) следует (14), так как максимум модуля $T_{n}^{(k)}(x)$ достигается только на конщах отрезка $[-1,1][4$, с. 286]. Если $x \in$ $\left[x_{n-k+1}, x_{1}\right]$, то из (13) следует (14).

При $k=1$

$$
\begin{aligned}
x_{i} & =\cos ((2 i-1) \pi /(2 n)), \quad i=\overline{1, n}, \\
T_{n}^{(1)}\left(x_{i}\right) & =(-1)^{i-1} n /\left(1-x_{i}^{2}\right)^{1 / 2} .
\end{aligned}
$$

При $k=2$

$$
\begin{aligned}
x_{i} & =\cos (i \pi / n), \quad i=\overline{1, n-1}, \\
T_{n}^{(2)}\left(x_{i}\right) & =(-1)^{i-1} n^{2} /\left(1-x_{i}^{2}\right) .
\end{aligned}
$$

Отсюда следует, что $c_{n, 1}=c_{n, 2}=1$ и $b_{n, 1} \leqslant n, b_{n, 2} \leqslant n^{2} / 4$.

При $k=n-1$

$$
T_{n}^{(n-2)}(x)=2^{n-2} n ! x^{2}-n 2^{n-3}(n-2) !, \quad x_{1}^{2}=x_{2}^{2}=1 /(2(n-1)) .
$$

Подставляя значение $x_{1}$ в (14), получим равенство

$$
m_{n, n-1}=(2(n-1))^{n / 2} /(2 n-3)^{(n-1) / 2} .
$$

При $k=n-2$

$$
\begin{aligned}
T_{n}^{(n-3)}(x) & =2^{n-1} n ! x^{3} / 3 !-n 2^{n-3}(n-2) ! x, \\
x_{1}^{2} & =x_{3}^{2}=3 /(2(n-1)), \quad x_{2}=0 .
\end{aligned}
$$


Подставив значения корней $x_{i}$ в (14), получим равенство

$$
m_{n, n-2}=((2 n-3) / 2)((2(n-1)) /(2 n-5))^{(n-2) / 2} .
$$

Докажем неравенства (16). При помоши тождества

$$
\left(1-x^{2}\right) T_{n}^{(k)}(x)-(2 k-3) x T_{n}^{(k-1)}(x)+\left(n^{2}-(k-2)^{2}\right) T_{n}^{(k-2)}(x) \equiv 0
$$

нетрудно показать, что в точках

$$
y_{i}^{(k)}=\cos ((k / 2+i-1) \pi / n)
$$

$$
z_{i}^{(k)}=\cos (((k+1) / 2+i-1) \pi / n)
$$

$(i=\overline{1, n-k})$ для полинома $T_{n}^{(k)}(x)(k=\overline{2,5})$ справедливы равенства

$$
\operatorname{sign} T_{n}^{(k)}\left(y_{i}^{(k)}\right)=(-1)^{i-1}, \quad \operatorname{sign} T_{n}^{(k)}\left(z_{i}^{(k)}\right)=(-1)^{i} .
$$

Отсюда следует, что для нулей $x_{i}^{(k)}$ полинома $T_{n}^{(k)}(x) \quad(k=\overline{2,5})$ справедливы неравенства

$$
\left|z_{i}^{(k)}\right|<\left|x_{i}^{(k)}\right|<\left|y_{i}^{(k)}\right|, \quad i=\overline{1, n-k} .
$$

Пусть $k=5$. Зафиксируем некоторое $i \in \overline{1, n-k}$. Для точки $x_{i}^{(5)}$ из (18) следует равенство

$$
\left(1-\left(x_{i}^{(5)}\right)^{2}\right) T_{n}^{(6)}\left(x_{i}^{(5)}\right)+\left(n^{2}-4^{2}\right) T_{n}^{(4)}\left(x_{i}^{(5)}\right)=0 .
$$

В силу доказанного выше на отрезке $\left[z_{i}^{(5)}, y_{i}^{(5)}\right]$ найдется точка $x_{i+1}^{(3)}$, для которой $T_{n}^{(3)}\left(x_{i+1}^{(3)}\right)=0$, и на этом отрезке точки $x_{i}^{(5)}$ и $x_{i+1}^{(3)}$ будут точками максимума функций $\left|T_{n}^{(4)}(x)\right|$ и $\left|T_{n}^{(2)}(x)\right|$ соответственно. Отсюда следуют неравенства

$$
\left|T_{n}^{(4)}\left(x_{i}^{(5)}\right)\right|>\left|T_{n}^{(4)}\left(x_{i+1}^{(3)}\right)\right|>\left(n^{2}-4\right)\left|T_{n}^{(2)}\left(z_{i}^{(5)}\right)\right| /\left(1-\left(x_{i+1}^{(3)}\right)^{2}\right) .
$$

Из теоремы о дифференциальных неравенствах [10, с. 15$]$ следует, что функция $\sin (a x) / \sin (b x)$ при $0<a<b$ на интервале $(0, \pi / 2 b)$ монотонно убывает вместе c $x$. Отсюда и из (19), (20) следует неравенство

$$
c_{n, 6} \geqslant \min _{i=\overline{1, n-5}}\left(\sin ^{4}(5 / 2+i-1) \pi / n\right) /\left(\sin ^{4}(3+i-1) \pi / n\right) \geqslant(5 / 6)^{4} \approx 0.482 .
$$

Аналогично доказывается, что $c_{n, 5} \geqslant(4 / 5)^{3}=0.512 ; c_{n, 4} \geqslant(3 / 4)^{2}=0.5625$; $c_{n, 3}>2 / 3$.

Докажем для $c_{n, 3}$ и $c_{n, 4}$ более точные оценки. Введем замену переменной $x=$ $\cos v, x \in[-1,1]$. Выпишем представления первых двух производных $T_{n}^{\prime}(x)$ и $T_{n}^{(2)}(x)$ через координату $v$ :

$$
\begin{aligned}
T_{n x}^{\prime}(\cos v) & =n \sin (n v) / \sin v \\
T_{n x^{2}}^{(2)}(\cos v) & =-n(n \cos (n v) \sin v-\sin (n v) \cos v) / \sin ^{3} v .
\end{aligned}
$$


Пусть $x_{i}^{(2)}=\cos v_{i}>0$ - нули полинома $T_{n}^{(2)}(x), i=\overline{1, i_{1}}, i_{1}=[(n-2) / 2]$, $x_{1}^{(2)}>x_{2}^{(2)}>\cdots>x_{i_{1}}^{(2)}$. Тогда из $(22)$ следует равенство

$$
n \operatorname{tg} v_{i}=\operatorname{tg}\left(n v_{i}\right), \quad i=\overline{1, i_{1}} .
$$

Если $v=\pi / 2$ - нуль полинома $T_{n x^{2}}^{(2)}(\cos v)$, то $n$ нечетно и $|\sin (n v)|=1$. Поэтому из (18) и (23) следует, что $c_{n, 3}=\left|\sin \left(n v_{1}\right)\right|$. Как показано выше, $v_{1} \in$ $(\pi / n, 3 \pi /(2 n))$. Из теоремы о дифференциальных неравенствах следует, что максимум функции $f(\alpha)=\cos (\alpha \pi) /(3 / 2-\alpha)$ на отрезке [0,1/2] реализуется в одной точке $\alpha_{0}$. Тогда для любого $n \geqslant 2 \quad v_{1}=\left(3 / 2-\alpha_{1}\right) \pi / n$, где $\alpha_{1} \leqslant \alpha_{0}$. В предположении противного существуют $n$ и $\alpha_{1}>\alpha_{0}, \alpha_{1} \in[0,1 / 2]$, для которых выполнены неравенства

$$
\begin{aligned}
\cos \left(\alpha_{0} \pi\right) / \cos \left(\alpha_{1} \pi\right) & <\sin \left(\left(3 / 2-\alpha_{0}\right) \pi / n\right) / \sin \left(\left(3 / 2-\alpha_{1}\right) \pi / n\right) \\
& <\left(3 / 2-\alpha_{0}\right) /\left(3 / 2-\alpha_{1}\right) .
\end{aligned}
$$

Но (24) противоречит определению $\alpha_{0}$. Следовательно, $c_{n, 3} \geqslant\left(\alpha_{0} \pi\right)$. Расчеты показывают, что $\alpha_{0} \approx 0.06970$ и $\cos \left(\alpha_{0} \pi\right) \geqslant 0.9761$.

Пусть $k=3$. Вычисления показьвают, что при $n \leqslant 8 c_{n, 4} \geqslant 0.9689$. Пусть $n \geqslant 9$ и $x_{i}^{(3)}=\cos v_{i}(i=\overline{1,[(n-3) / 2]})$ - положительные корни полинома $T_{n}^{(3)}(x)$. Тогда из (18) для $v_{i}$ следует равенство

$$
3 \sin ^{2} v_{i} T_{n x^{2}}^{(2)}\left(\cos v_{i}\right)=\left(n^{2}-1\right) \sin \left(n v_{i}\right) \operatorname{tg} v_{i} .
$$

Для $v_{i}$ имеем представление $v_{i}=\left(i+1-\alpha_{i}\right) \pi / n$, где $\alpha_{i} \in(0,1 / 2)$. При $i<j$ $\operatorname{tg} v_{i}<\operatorname{tg} v_{j}$, и если при этом $\alpha_{j} \geqslant \alpha_{i}$, то имеют место неравенства

$$
\left|\sin \left(n v_{j}\right)\right|>\left|\sin \left(n v_{i}\right)\right|, \quad\left|\left(1-x_{j}^{2}\right) T_{n}^{(2)}\left(x_{j}\right)\right|>\left|\left(1-x_{i}^{2}\right) T_{n}^{(2)}\left(x_{i}\right)\right| .
$$

Если $T_{n}^{(3)}(x)$ имеет корень $x=0$, то $n$ четно и $\left|T_{n}^{(2)}(0)\right|=n^{2}$.

Рассмотрим функции

$$
\begin{aligned}
& \varphi(\alpha, p)=(\cos (\alpha \pi)+\sin (\alpha \pi) /(\pi(p-\alpha))) /(p-\alpha)^{2} \\
& \psi(\alpha, p)=\frac{\cos (\alpha \pi)+\sin (\alpha \pi) \sum_{j=0}^{3}\left((-1)^{j}((p-\alpha) \pi / 9)^{2 j} /(2 j) !\right) /((p-\alpha) \pi)}{(p-\alpha)^{2}},
\end{aligned}
$$

где $p=2,3, \ldots ; \alpha \in[0,1 / 2]$.

На каждом отрезке $[p-1 / 2, p] \pi / n, p=\overline{2,[(n-3) / 2]+1}$, имеют место неравенства

$$
(p-\alpha)^{2} \varphi(\alpha, p) \geqslant\left|\sin ^{2} v T_{n x^{2}}^{(2)}(\cos v) / n^{2}\right| \geqslant(p-\alpha)^{2} \psi(\alpha, p),
$$

где $v=(p-\alpha) \pi / n$.

Зафиксируем $p$. Из теоремы о дифференциальных неравенствах следует, что функции $\varphi(\alpha, p)$ и $\psi(\alpha, p)$ имеют на отрезке $[0,1 / 2]$ по одному локальному максимуму соответственно в точках $\alpha_{1}^{(p)}$ и $\alpha_{2}^{(p)}$. Пусть $\alpha_{3}^{(p)}$ минимальная из $\alpha \in[0,1 / 2]$, для которой выполнены неравенства

$$
\max \left(\alpha_{1}^{(p)}, \alpha_{2}^{(p)}\right) \leqslant \alpha_{3}^{(p)}, \quad \varphi\left(\alpha_{3}^{(p)}, p\right) \leqslant \psi\left(\alpha_{2}^{(p)}, p\right) .
$$


Тогда из $(27)$ вытекают неравенства $\alpha_{p-1} \leqslant \alpha_{3}^{(p)}$ и

$$
\begin{aligned}
c_{n, 4} & \geqslant \min _{p} \frac{\psi\left(\alpha_{2}^{(p)}, p\right)\left(p-\alpha_{2}^{(p)}\right)^{2} \sin ^{2}\left(\left(p-\alpha_{3}^{(p)}\right) \pi / n\right)}{\sin ^{2}\left(\left(p-\alpha_{2}^{(p)}\right) \pi / n\right)} \\
& \geqslant \min _{p} \psi\left(\alpha_{2}^{(p)}, p\right)\left(p-\alpha_{3}^{(p)}\right)^{2}
\end{aligned}
$$

Вычисления показывают, что

$$
\begin{aligned}
& \alpha_{3}^{(2)} \approx 0.22344, \quad \psi\left(\alpha_{2}^{(2)}, 2\right)\left(2-\alpha_{3}^{(2)}\right)^{2} \geqslant 0.879, \\
& \alpha_{3}^{(3)} \approx 0.16061, \quad \psi\left(\alpha_{2}^{(3)}, 3\right)\left(3-\alpha_{3}^{(3)}\right)^{2} \geqslant 0.929 .
\end{aligned}
$$

При $p \geqslant 4$

$$
\psi(0, p)\left(p-\alpha_{3}^{(3)}\right)^{2}=\left(p-\alpha_{3}^{(3)}\right)^{2} / p^{2} \geqslant 0.915
$$

Из (29) и (26) следует неравенство $c_{n, 4} \geqslant 0.879$.

Из оценок сверху для $x_{1}^{(k)}, k=\overline{1,5}$, вытекают оценки сверху для $b_{n, k+1}$ :

$$
\begin{aligned}
& b_{n, n}=1 ; \quad \text { при } n \geqslant k+1 \quad b_{n, 3} \leqslant n^{3} /(3.6)^{3} ; \quad b_{n, 4} \leqslant n^{4} /(4.49)^{4} \text {; } \\
& b_{n, 5} \leqslant n^{5} /(5.19)^{5} ; \quad b_{n, 6} \leqslant n^{6} /(6.3)^{6} \text {. }
\end{aligned}
$$

Отсюда следует, что при $k=\overline{1,6} m_{n, k}=T_{n}^{(k)}(1) / a_{n, k}$. Лемма доказана.

\section{§3. Оценки норм производных полиномов на кубе в $\mathbb{R}^{m}$}

Введем обозначение $Q^{m}=\left\{x \in \mathbb{R}^{m}:\left|x_{i}\right| \leqslant 1, i=\overline{1, m}\right\}$.

Tеорема 1. Пусть $X=\ell_{2}^{(m)}, \quad P_{n}(x) \in \mathfrak{P}_{n}(X, \mathbb{R}) u\left\|P_{n}\right\|_{Q^{m}} \leqslant 1$. Тогдa справедливы неравенства

$$
\begin{aligned}
\left\|\nabla P_{n}\right\|_{Q^{m}} & \leqslant n^{2}, \\
\left\|\nabla^{2} P_{n}\right\|_{Q^{m}} & \leqslant A=n^{2}(n-1) \min (2(n+1) / 3, n-1) .
\end{aligned}
$$

ДокАЗАТЕльство. Для доказательства неравенства (30) потребуется обобшенное неравенство Бернштейна, полученное в работе [11]. Приведем его формулировку.

Пусть $V$ - центрально-симметричный выпуклый компакт в $\ell_{2}^{(m)}, V^{*}$ - поляра множества $V$. Для $z=\left(z_{1}, z_{2}, \ldots, z_{m}\right) \in \mathbb{C}^{m}$ положим

$$
\|z\|_{V}=\sup _{t \in V^{*}}\left|\sum_{i=1}^{m} t_{i} z_{i}\right| .
$$

Целая функция $f(z)$ имеет степень $V$, если для каждого $\varepsilon>0$ найдется $A_{\varepsilon}$ такое, чTO

$$
|f(z)| \leqslant A_{\varepsilon} \exp \left\{(1+\varepsilon)\|z\|_{V^{*}}\right\}
$$


для каждого $z \in \mathbb{C}^{m}$. Множество всех целых функций степени $V$, ограниченных на $\mathbb{R}^{m}$, обозначается $B(V)$. Пусть $\sigma>0$ и $g \in B(\sigma V)$. Тогда имеет место неулучшаемое неравенство

$$
\sup _{x \in \ell_{2}^{(m)}}\|\nabla g(x)\|_{\ell_{2}^{(m)}} \leqslant(\sigma \operatorname{diam}(V) / 2) \sup _{x \in \ell_{2}^{(m)}}|g(x)|,
$$

где $\operatorname{diam}(V)=\max _{x, y \in V}\|x-y\|_{\ell_{2}^{(m)}}$.

Введем замену переменных $x_{i} \stackrel{2}{=} \cos v_{i}$. Нетрудно проверить, что тригонометрический полином $P_{n}\left(\cos v_{1}, \ldots, \cos v_{m}\right)$ является целой функцией степени $n E$, где $E$ - шар радиуса единица в пространстве $\ell_{1}^{(m)}: E=\left\{v \in \mathbb{R}^{m}: \sum_{i=1}^{m}\left|v_{i}\right| \leqslant 1\right\}$. Диаметр шара $E$ в метрике $\ell_{2}^{(m)}$ равен 2 , поэтому из $(32)$ для полинома $P_{n}(x)$ следует неравенство

$$
\left|\sum_{i=1}^{m}\left(1-x_{i}^{2}\right)^{1 / 2} P_{n x_{i}}^{\prime}(x) h_{i}\right| \leqslant n
$$

где $x \in Q^{m}, h=\left(h_{1}, \ldots, h_{m}\right) \in \ell_{2}^{(m)},\|h\| \leqslant 1$.

Пусть $\hat{x}=\left(\hat{x}_{1}, \hat{x}_{2}, \ldots, \hat{x}_{m}\right) \in Q^{m}$ и $\left|\hat{x}_{i_{1}}\right|=\max _{1 \leqslant i \leqslant m}\left|\hat{x}_{i}\right|$. Пусть $d-$ отрезок прямой $\left\{k_{1} x_{i_{1}}, k_{2} x_{i_{1}}, \ldots, k_{m} x_{i_{1}}\right\}, x_{i_{1}} \in[-1,1]$, проходящий через точки 0 и $\hat{x}$ $\left(k_{i}=\hat{x}_{i} / \hat{x}_{i_{1}}\right)$ и принадлежаший $Q^{m}$. Для $x \in d\left|x_{i}\right| \leqslant\left|x_{i_{1}}\right|, i=\overline{1, m}$, поэтому, подставив в (33) вектор $h^{1}\left(h_{i}^{1}=h_{i}\left(\left(1-x_{i_{1}}^{2}\right) /\left(1-x_{i}^{2}\right)\right)^{1 / 2}, i=\overline{1, m}\right)$, получим неравенство

$$
\left(1-x_{i_{1}}^{2}\right)^{1 / 2}\left|\left(\nabla P_{n}(x), h\right)\right| \leqslant n, \quad x \in d .
$$

В силу леммы 2 из (34) следует (30).

Докажем неравенство (31). Пусть $U_{n}\left(v_{1}, \ldots, v_{m}\right)=P_{n}\left(\cos v_{1}, \ldots, \cos v_{m}\right)$. Тогда для любого нормированного вектора $h \in \ell_{2}^{(m)}$ справедливы неравенства

$$
\begin{array}{r}
|(\nabla U(v), h)| \leqslant n, \\
\left\|\nabla^{2} U(v) h\right\|_{\ell_{2}^{(m)}} \leqslant n^{2},
\end{array}
$$

которые вытекают из неравенства (32). Пусть $A(x)=D \nabla^{2} P_{n}(x) D$, где $D$ - диагональная матрица с элементами $\sin v_{i}(i=\overline{1, m})$ по диагонали. Вследствие (36) и (30) имеем неравенство

$$
\|A(x)\|_{\ell_{2}^{(m)}} \leqslant 2 n^{2}, \quad x \in Q^{m} .
$$

Пусть $D_{1}$ - диагональная матрица с элементами $\sin v_{i_{1}} / \sin v_{i}(i=\overline{1, m})$. Тогда для точек отрезка $d \subset Q^{m}$ имеем неравенство

$$
\left\|\sin ^{2} v_{i_{1}} \nabla^{2} P_{n}(x)\right\|_{\ell_{2}^{(m)}}=\left\|D_{1} A(x) D_{1}\right\|_{\ell_{2}^{(m)}} \leqslant 2 n^{2}, \quad x \in d .
$$

Пусть $h$ и $h_{1}-$ произвольные нормированные векторы из $\ell_{2}^{(m)}$. Тогда для полинома $\left(\nabla^{2} P_{n}(x) h, h_{1}\right)$ на отрезке $d$ справедливо неравенство

$$
\left|\left(1-x_{i_{1}}^{2}\right)\left(\nabla^{2} P_{n}(x) h, h_{1}\right)\right| \leqslant 2 n^{2} .
$$

Из (38) и леммы 2 вытекает неравенство (31). Теорема доказана. 
СлЕДСТВИЕ 1. Пусть выполнены условия теоремы 1. Тогда справедливы неравенства

$$
(1 / 3)^{p} \prod_{i=0}^{p-1}(n-2 i)^{2}\left((n-2 i)^{2}-1\right)=M_{2 p}
$$

npu $k=2 p$

$$
\left\|\nabla^{k} P_{n}\right\|_{Q^{m}} \leqslant N_{2 p}=\min _{0 \leqslant i \leqslant p}\left\{2^{p-i} M_{2(p-i)} \prod_{j=2 i}^{1}\left(n-(k-j)^{2}\right)\right\},
$$

$n p u k=2 p+1$

$$
\left\|\nabla^{k} P_{n}\right\|_{Q^{m}} \leqslant N_{2 p}(n-2 p)^{2}, \quad p \geqslant 1 .
$$

Доказательство следует из последовательного применения теоремы 1 к полиному $\left\langle\nabla^{k} P_{n}(x),\left[h_{1}, h_{2}, \ldots, h_{k}\right]\right\rangle$, его градиенту $\left\langle\nabla^{k+1} P_{n}(x),\left[h_{1}, h_{2}, \ldots, h_{k}\right]\right\rangle$ или гессиану $\left\langle\nabla^{k+2} P_{n}(x),\left[h_{1}, h_{2}, \ldots, h_{k}\right]\right\rangle$. Здесь $h_{j}(j=\overline{1, k})$ - произвольные фиксированные нормированные векторы из $\ell_{2}^{(m)}$.

СЛЕДСТВИЕ 2. Пусть $U-$ линейный оператор из $\ell_{2}^{(m)}{ }_{\text {в }} \ell_{2}^{(m)}, \quad T=U Q$, $y=U x, \quad x \in Q^{m}$. Пусть $B_{n}(y) \in \mathfrak{P}_{n}(X, \mathbb{R}) u\left\|B_{n}\right\|_{T} \leqslant 1$.

Тогда для полинома $B_{n}(y)$ при $y \in T$ справедливо неравенство

$$
\left\|U^{*} \nabla B_{n}(y)\right\|_{\ell_{2}^{(m)}} \leqslant n^{2},
$$

и если U невырождено, то

$$
\left\|\nabla B_{n}(y)\right\|_{\ell_{2}^{(m)}} \leqslant\left(\min _{1 \leqslant j \leqslant m} \lambda_{j}\right)^{-1 / 2} n^{2},
$$

где $\lambda_{j}-$ собственные значения матрицы $U U^{*}$.

Подобные неравенства можно распространить и на последуюшие производные, учитывая следствие 1.

Пусть на $\mathbb{R}^{m}$ определена некоторая норма $\|\cdot\|$. Пусть

$$
\begin{aligned}
S_{n}(x) & =\sin (\operatorname{narccos} x), \quad x \in[-1,1], \quad k, j=\overline{1, n}, \quad i=(-1)^{1 / 2}, \\
B_{n}^{j} & =\max _{x \in[-1,1]}\left(1-x^{2}\right)^{j / 2} \min \left\{\left|T_{n}^{(j)}(x)+i S_{n}^{(j)}(x)\right|, T_{n}^{(j)}(1)\right\}, \\
A_{n}^{j, k} & =\max _{x \in\left[x_{n-k+1}^{(k-1)}, x_{1}^{(k-1)}\right]}\left(1-x^{2}\right)^{j / 2}\left|T_{n}^{(j)}(x)+i S_{n}^{(j)}(x)\right|,
\end{aligned}
$$

где $x_{1}^{(k-1)} \geqslant x_{2}^{(k-1)} \geqslant \cdots \geqslant x_{n-k+1}^{(k-1)}-$ корни полинома $T_{n}^{(k-1)}(x)$.

TeOPEMA 2. Пусть $X=\mathbb{R}^{m}, \quad P_{n}(x) \subset \mathfrak{P}_{n}(X, \mathbb{R}), \quad\left\|P_{n}\right\|_{Q^{m}} \leqslant 1$. Пусть $k=\sum_{i=1}^{p} k_{i}$, дде $k_{i} \in \mathbb{Z}_{+}, k_{1} \leqslant k_{2} \leqslant \cdots \leqslant k_{p}$. Тогда справедливо неравенство

$$
\begin{aligned}
\max _{x \in Q^{m}}\left|\frac{\partial^{k} P_{n}(x)}{\partial x_{j_{1}}^{k_{1}} \ldots \partial x_{j_{p}}^{k_{p}}}\right| & \leqslant B_{n}^{k_{1}} B_{n-k_{1}}^{k_{2}} \ldots B_{n-\sum_{j=1}^{p-2} k_{j}}^{k_{p-1}} A_{n-\sum_{j=1}^{p-1} k_{j}}^{k_{p}, k} m_{n, k} \\
& =C\left(n, k, k_{1}, \ldots, k_{p}\right),
\end{aligned}
$$

әде $m_{n, k}$ определено в лемме 2. 
ДокАЗАТЕЛЬСТВО. Пусть $\hat{x}=\left(\hat{x}_{1}, \ldots, \hat{x}_{m}\right) \in Q^{m}$ и $\left|\hat{x}_{i_{1}}\right|=\max \left|x_{i}\right| \quad(i=$ $\overline{1, m})$. Пусть $d$ - отрезок прямой $\left\{k_{1} x_{i_{1}}, \ldots, k_{m} x_{i_{1}}\right\} \quad\left(k_{i}=\hat{x}_{i} / \hat{x}_{i_{1}}, x_{i_{1}} \in[-1,1]\right)$, принадлежаший $Q^{m}$. Рассмотрим неравенство, полученное в работе [12]; если $\left\|P_{n}\right\|_{[-1,1]} \leqslant 1$, то

$$
\left|P_{n}^{(j)}(x)\right| \leqslant\left|T_{n}^{(j)}(x)+i S_{n}^{(j)}(x)\right|, \quad-1 \leqslant x \leqslant 1, \quad j=\overline{1, n} .
$$

Из (40) вытекает очевидное неравенство для $x \in[-1,1]$ :

$$
\left(1-x^{2}\right)^{j / 2}\left|P_{n}^{(j)}(x)\right| \leqslant\left(1-x^{2}\right)^{j / 2} \min \left\{\left|T_{n}^{(j)}(x)+i S_{n}^{(j)}(x)\right|, T_{n}^{(j)}(1)\right\} \leqslant B_{n}^{j} .
$$

Применяя последовательно $p-1$ раз неравенство (41) и один раз неравенство (40), получим для точек $x$, принадлежаших отрезку $d$,

$$
\max _{x_{n-k+1}^{(k-1)} \leqslant x_{i_{1}} \leqslant x_{1}^{(k-1)}}\left(1-x_{i_{1}}^{2}\right)^{k / 2}\left|\frac{\partial^{k} P_{n}(x)}{\partial x_{j_{1}}^{k_{1}} \ldots \partial x_{j_{p}}^{k_{p}}}\right| \leqslant B_{n}^{k_{1}} \ldots B_{n-\sum_{j=1}^{p-2} k_{j}}^{k_{p-1}} A_{n-\sum_{j=1}^{k_{p}, k} k_{j}}^{k_{1}} .
$$

Из (42) и леммы 2 следует (39). Теорема доказана.

СлЕДСТВИЕ 3. Пусть $X=\ell_{1}^{(m)}, \quad P_{n}(x) \in \mathfrak{P}_{n}(X, \mathbb{R}) u\left\|P_{n}\right\|_{Q^{m}} \leqslant 1$. Тогда имеет место неравенство при $k=\overline{2, n}$

$$
\begin{aligned}
\left\|\nabla^{k} P_{n}\right\|_{Q^{m}} & \leqslant \max \left\{T_{n}^{(k)}(1), \max C\left(n, k, k_{1}, \ldots, k_{p}\right)\right\}, \\
p & =\overline{2, k}, \quad k=\sum_{j=1}^{p} k_{j}, \quad k_{j} \in \mathbb{Z}_{+} .
\end{aligned}
$$

СлеДСТвИЕ 4. Пусть $X=\mathbb{R}^{m}, \quad P_{n}(x) \in \mathfrak{P}_{n}(X, \mathbb{R}), \quad \Pi=\left\{x:\left|x_{i}\right| \leqslant a_{i}\right.$, $i=\overline{1, m}\}, \quad\left\|P_{n}\right\|_{\Pi} \leqslant 1$. Тогда справедливо неравенство

$$
\max _{x \in \Pi}\left|\frac{\partial^{k} P_{n}(x)}{\partial x_{j_{1}}^{k_{1}} \ldots \partial x_{j_{p}}^{k_{p}}}\right| \leqslant\left|\frac{C\left(n, k, k_{1}, \ldots, k_{p}\right)}{a_{j_{1}}^{k_{1}} \ldots a_{j_{p}}^{k_{p}}}\right|, \quad k=\overline{2, n}, \quad p \geqslant 2 .
$$

ЗАМЕчАнИЕ. При небольших $k(k \leqslant 6)$ и $k=n-2, p=n-2 ; k=n-1, p=$ $n-1 ; k=n, p=n$ значения $C\left(n, k, k_{1}, \ldots, k_{p}\right)$ достаточно просто вычисляются. Заметим, что

$$
\begin{aligned}
\left|T_{n}^{(1)}(x)+i S_{n}^{(1)}(x)\right|= & n / \sin v \\
\left|T_{n}^{(2)}(x)+i S_{n}^{(2)}(x)\right|= & \left(n^{2} / \sin ^{2} v\right)\left(1+\operatorname{ctg}^{2} v / n^{2}\right)^{1 / 2}, \\
\left|T_{n}^{(3)}(x)+i S_{n}^{(3)}(x)\right|= & \left(n\left(n^{2}-1\right) / \sin ^{3} v\right) \\
& \times\left(1+\left(3\left(n^{2}+2\right) \operatorname{ctg}^{2} v+9 \operatorname{ctg}^{4} v\right) /\left(n^{2}-1\right)^{2}\right)^{1 / 2}
\end{aligned}
$$

где $x=\cos v$. 
Вьпишем значения и оценки $C\left(n, k, k_{1}, \ldots, k_{p}\right)$ для конкретных значений $k$, $k_{1}, \ldots, k_{p}$ :

$$
\begin{aligned}
& C(n, n, 1, \ldots, 1)=n !, \quad p=n ; \\
& C(n, n-1,1, \ldots, 1)=n !(2(n-1))^{n / 2} /(2 n-3)^{(n-1) / 2}, \quad p=n-1 ; \\
& C(n, n-2,1, \ldots, 1)=n !((2 n-3) / 4)(2(n-1) /(2 n-5))^{(n-2) / 2}, \quad p=n-2 ; \\
& k=2: \\
& C(n, 2,1,1)=n(n-1)\left(n^{2}-1\right) / 3 ; \\
& k=3: D=n^{2}(n-1)\left(n^{2}-2^{2}\right) /(3 \cdot 5), \\
& C(n, 3,1,1,1) \leqslant 1.0245(n-2) D, \quad C(n, 3,1,2) \leqslant 1.0801(n-1) D ; \\
& k=4: D=n\left(n^{2}-1\right)\left(n^{2}-3^{2}\right) /(3 \cdot 5 \cdot 7), \\
& C(n, 4,1,1,1,1) \leqslant 1.1377(n-1)(n-2)(n-3) D ; \\
& C(n, 4,1,1,2) \leqslant 1.2085(n-1)(n-2)^{2} D ; \\
& C(n, 4,1,3) \leqslant 1.2861(n-1)\left((n-1)^{2}-1\right) D ; \\
& C(n, 4,2,2) \leqslant 1.3693 n(n-2)^{2} D \text {; } \\
& k=5: D=n^{2}\left(n^{2}-2^{2}\right)\left(n^{2}-4^{2}\right) /(3 \cdot 5 \cdot 7 \cdot 9), \\
& C(n, 5,1,1,1,1,1) \leqslant 1.9532(n-1)(n-2)(n-3)(n-4) D ; \\
& C(n, 5,1,1,1,2) \leqslant 2.1021(n-1)(n-2)(n-3)^{2} D ; \\
& C(n, 5,1,1,3) \leqslant 2.2721(n-1)(n-2)\left((n-2)^{2}-1\right) D ; \\
& C(n, 5,1,2,2) \leqslant 2.3818(n-1)^{2}(n-3)^{2} D ; \\
& C(n, 5,2,3) \leqslant 2.5778 n(n-2)\left((n-2)^{2}-1\right) D \\
& C(n, 5,1,4) \leqslant 3.1568(n-1)^{2}\left((n-1)^{2}-1\right) D \text {; } \\
& k=6: D=n\left(n^{2}-1\right)\left(n^{2}-3^{2}\right)\left(n^{2}-5^{2}\right) /(3 \cdot 5 \cdot 7 \cdot 9 \cdot 11), \\
& C(n, 6,1,1,1,1,1,1) \leqslant 2.0736(n-1)(n-2)(n-3)(n-4)(n-5) D ; \\
& C(n, 6,1,1,1,1,2) \leqslant 2.2198(n-1)(n-2)(n-3)(n-4)^{2} D ; \\
& C(n, 6,1,1,1,3) \leqslant 2.3838(n-1)(n-2)(n-3)\left((n-3)^{2}-1\right) D \text {; } \\
& C(n, 6,1,1,2,2) \leqslant 2.5151(n-1)(n-2)^{2}(n-4)^{2} D ; \\
& C(n, 6,1,2,3) \leqslant 2.4115(n-1)^{2}(n-3)\left((n-3)^{2}-1\right) D ; \\
& C(n, 6,2,2,2) \leqslant 2.8556 n(n-2)^{2}(n-4)^{2} D ; \\
& C(n, 6,3,3) \leqslant 3.0590\left(n^{2}-1\right)(n-3)\left((n-3)^{2}-1\right) D ; \\
& C(n, 6,2,4) \leqslant 3.3043 n(n-2)^{2}\left((n-2)^{2}-1\right) D \\
& C(n, 6,1,1,4) \leqslant 3.2664(n-1)(n-2)^{2}\left((n-2)^{2}-1\right) D \\
& C(n, 6,1,5) \leqslant 4.03(n-1)\left((n-1)^{2}-1\right)\left((n-1)^{2}-6\right) D \text {. }
\end{aligned}
$$




\section{$\S 4$. Оценки норм производных полиномов на выпуклых телах в банаховом пространстве}

Далее $X$ - банахово пространство.

TeOpema 3. Пусть $K \subset X, \quad P_{n}(x) \in \mathfrak{P}_{n}(X, \mathbb{R}), \quad\left\|P_{n}\right\|_{K} \leqslant 1$. Тогда для любого нормированного вектора $h \in X$ выполнено неравенство

$$
\begin{gathered}
\left|\left\langle\nabla P_{n}(x), h\right\rangle\right| \leqslant \inf _{y_{1}, y_{2} \in K}\left(2 n^{2} /\left\|y_{1}-y_{2}\right\|\right)\left(\left\|x-y_{1}\right\| /\left\|y_{1}^{1}-y_{1}^{2}\right\|\right. \\
\left.\quad+\left\|x-y_{2}\right\| /\left\|y_{2}^{1}-y_{2}^{2}\right\|\right) \leqslant 4 n^{2} / \omega_{K}(h) \\
\leqslant 4 n^{2} / \omega_{K} \leqslant 2 n^{2} / r(K),
\end{gathered}
$$

əде $x, y_{1}, y_{2} \in K, \quad y_{1}-y_{2}=c h, y_{i}^{j} \in \partial K \quad(i, j=1,2), y_{i}, x \in\left[y_{i}^{1}, y_{i}^{2}\right] \quad(i=1,2)$, $\omega_{K}(h)=\sup _{y_{1}, y_{2}}\left\|y_{1}-y_{2}\right\|, \omega_{K}=\inf _{\|h\|=1} \omega_{K}(h)$.

ДоКАЗАТЕЛЬСТВО. Пусть $y_{1}, y_{2} \in K \quad y_{1}-y_{2}=c h$. Тогда для произвольной точки $x \in K$ имеем неравенство

$$
\left|\left\langle\nabla P_{n}(x), y_{1}-y_{2}\right\rangle\right| \leqslant\left|\left\langle\nabla P_{n}(x), x-y_{1}\right\rangle\right|+\left|\left\langle\nabla P_{n}(x), x-y_{2}\right\rangle\right| .
$$

Разделив обе части неравенства (44) на $\left\|y_{1}-y_{2}\right\|$ и применив в оценке двух членов правой части неравенство (1), получим неравенство (43). Теорема доказана.

СлЕДСТвИЕ 5. При условиях теоремы 3 выполнено неравенство

$$
\left\|\nabla^{k} P_{n}\right\|_{K} \leqslant\left(4 / \omega_{k}\right)^{k} n^{2}(n-1)^{2} \ldots(n-(k-1))^{2} .
$$

СлеДСТВИЕ 6. Пусть К является т-мерным симплексом. Если $\left|P_{n}(x)\right| \leqslant 1$ при $x \in K$, то справедливо неравенство

$$
\left\|\nabla P_{n}(x)\right\|_{K} \leqslant 4 n^{2} / \min h_{i}, \quad i=\overline{1, m+1},
$$

где $h_{i}$ - минимальное расстояние между $i$-й вершиной и противоположной гранью симплекса.

Для правильного т-мерного симплекса с ребром 1 в метрике $\ell_{2}^{(m)}$ получим оченку

$$
\max _{x \in K}\left\|\nabla P_{n}(x)\right\|_{\ell_{2}^{(m)}} \leqslant 4 n^{2} \sqrt{2 m /(m+1)} .
$$

Для правильного двумерного симплекса $K$ с ребром 1 в метрике $\ell_{2}^{(2)}$ и основанием, параллельныц оси $0 x_{1}$, для пространства $\ell_{\infty}^{(2)}$ получим оценку

$$
\max _{x \in K}\left\|\nabla P_{n}(x)\right\|_{\ell_{1}^{(2)}} \leqslant \frac{8 n^{2}}{3-\sqrt{3}}<\frac{12 n^{2}}{\sqrt{3}}<\frac{4 n^{2}}{2 \sqrt{3}-3},
$$

где $\sqrt{3} / 6, \quad(2 \sqrt{3}-3) / 2$ - радиусы максимальных шаров, содержсащихся в $K$, в метрике $\ell_{2}^{(2)}$ и $\ell_{\infty}^{(2)}$ соответственно.

Teopema 4. Пусть $K \subset X, P_{n} \in \mathfrak{P}_{n}(X, \mathbb{R}),\left\|P_{n}\right\|_{K} \leqslant 1, x \in K \backslash \partial K$. Тогда

$$
\left\|\nabla P_{n}(x)\right\|_{\mathscr{L}(X, \mathbb{R})} \leqslant b_{1}(x) n \leqslant \sqrt{2} b(x) n .
$$


ДоказАтельство. Пусть $x_{0}, x \in K \backslash \partial K, h \in X$ и $\|h\|=1$. Пусть $\tilde{x} \in$ $\partial K, x \in\left[x_{0}, \tilde{x}\right]$, отрезок $\left[y_{1}, y_{2}\right]$ является диаметром шара с центром в точке $x_{0}$ и $\left\|y_{1}-x_{0}\right\|=r_{1}\left(x_{0}\right) \geqslant r\left(x_{0}\right)-\varepsilon(\varepsilon>0), y_{1}-y_{2}=c h$. Пусть в точках $y_{1}, y_{2}, \tilde{x}$ сосредоточены соответственно массы $1,1,2 \rho\left(x, x_{0}\right) /\left(1-\rho\left(x, x_{0}\right)\right)$.

В силу определения $\rho\left(x, x_{0}\right)=\rho$ имеем равенство

$$
\left\|\tilde{x}-x_{0}\right\| \rho\left(x, x_{0}\right)=\left\|x-x_{0}\right\|,
$$

откуда следует, что точка $x$ является центром тяжести системы материальных точек $\left(y_{1}, 1\right),\left(y_{2}, 1\right),(\tilde{x}, 2 \rho /(1-\rho))$.

Пусть прямая, содержащая точки $y_{1}$ и $x$, пересекает отрезок $\left[y_{2}, \tilde{x}\right]$ в точке $x_{1}$. Аналогично определим точку $x_{2}$. Точки $x_{1}$ и $x_{2}$ являются центрами тяжести систем материальных точек $\left(y_{2}, 1\right),(\tilde{x}, 2 \rho /(1-\rho))$ и $\left(y_{1}, 1\right),(\tilde{x}, 2 \rho /(1-\rho))$ соответственно. Отсюда следуют равенства

$$
(1-\rho)\left\|x-y_{i}\right\|=(1+\rho)\left\|x_{i}-x\right\|, \quad i=1,2 .
$$

Из (47) и (2) вытекают равенства

$$
\left|\left(\nabla P_{n}(x), y_{i}-x\right)\right| \leqslant n \sqrt{1+\rho} / \sqrt{1-\rho}, \quad i=1,2 .
$$

Так как $y_{1}-y_{2}=\left(y_{1}-x\right)-\left(y_{2}-x\right)=2 h r_{1}\left(x_{0}\right)$, то из (48) следует (45).

ТЕОРема 5. Пусть $K$ - иентрально-симметричное ограниченное замкнутое выпуклое тело из $X$. Пусть $P_{n}(x) \in \mathfrak{P}_{n}(X, \mathbb{R}) u\left\|P_{n}\right\|_{K} \leqslant 1$.

Тогда справедливы неравенства

$$
\begin{aligned}
\left\|\nabla P_{n}\right\|_{K} & \leqslant B_{1} / r(K) \leqslant \sqrt{2} n^{2} / r(K), \\
\left\|\nabla P_{n}(x)\right\|_{\mathscr{L}(X, \mathbb{R})} & \leqslant \sqrt{2} n /\left(r(K)\left(1-\rho^{2}\left(x, x_{0}\right)\right)^{1 / 2}\right), \quad x \in K \backslash \partial K, \\
\sup _{x \in K}\left|\left\langle\nabla^{2} P_{n}(x) h, h\right\rangle\right| & \leqslant C_{1} / r^{2}(K) \leqslant C_{2} / r^{2}(K),
\end{aligned}
$$

әде $x_{0}$ - центр симметрии $K,\|h\| \leqslant 1$.

ДоКАЗАТЕЛЬСТво. В силу выпуклости $K$ точка $x_{0}$ будет центром шара $B\left(x_{0}, r(K)\right) \subset K$. Пусть $x \in K$ и $\left[y_{1}, y_{2}\right]$ - некоторый диаметр шара $B\left(x_{0}, r(K)\right)$. Положим $u_{1}=\left(y_{1}-x\right), u_{2}=\left(y_{2}-x\right)$.

В силу центральной симметрии тела $K$ в нем содержится параллелограмм $P$ с центром в $x_{0}$, одна из вершин параллелограмма есть точка $x$, а стороны совпадают с векторами $u_{1}$ и $u_{2}$. Каждая точка $y \in P$ представляется в виде

$$
y=x_{0}-\left(t_{1} u_{1}+t_{2} u_{2}\right) / 2, \quad\left|t_{i}\right| \leqslant 1 .
$$

Полином $U_{n}(t)=P_{n}\left(x_{0}-\left(t_{1} u_{1}+t_{2} u_{2}\right) / 2\right)$ является полиномом $n$-й степени от двух переменных $t_{1}$ и $t_{2}$. Применяя теорему 1 , получим неравенство

$$
\begin{aligned}
\left|\left\langle\nabla P_{n}(x), y_{1}-y_{2}\right\rangle\right| / 2 & =\left|\left\langle\nabla P_{n}(x), u_{1}\right\rangle-\left\langle\nabla P_{n}(x), u_{2}\right\rangle\right| / 2 \\
& =\left|U_{n t_{1}}^{\prime}\left(t_{1}, t_{2}\right)-U_{n t_{2}}^{\prime}\left(t_{1}, t_{2}\right)\right|_{t_{1}=t_{2}=1} \leqslant \sqrt{2} n^{2} .
\end{aligned}
$$


Из (52) следует (49). В силу теоремы 1 имеет место неравенство

$$
\begin{gathered}
\left|\left\langle\nabla^{2} P_{n}(x)\left(y_{1}-y_{2}\right), y_{1}-y_{2}\right\rangle\right| / 4=\mid\left\langle\nabla^{2} P_{n}(x) u_{1}, u_{1}\right\rangle+\left\langle\nabla^{2} P_{n}(x) u_{2}, u_{2}\right\rangle \\
\quad-2\left\langle\nabla^{2} P_{n}(x) u_{1}, u_{2}\right\rangle|/ 4=|\left\langle\nabla^{2} U_{n}(1,1)(1,-1)^{T},(1,-1)^{T}\right\rangle \mid \\
\leqslant 2\left\|\nabla^{2} U_{n}(1,1)\right\|_{\ell_{2}^{(2)}} \leqslant 2 n^{2}(n-1) \min (2(n+1) / 3, n-1) .
\end{gathered}
$$

С другой стороны, в силу теоремы 2 имеем неравенство

$$
\left|\left\langle\nabla^{2} P_{n}(x) u_{1}, u_{2}\right\rangle\right| / 4=\left|U_{n t_{1} t_{2}}^{\prime \prime}(1,1)\right| \leqslant n(n-1)\left(n^{2}-1\right) / 3 .
$$

Из (1) и (54) вытекает неравенство

$$
\left|\left\langle\nabla^{2} P_{n}(x)\left(y_{1}-y_{2}\right), y_{1}-y_{2}\right\rangle\right| / 4 \leqslant 2 n(2 n-1)\left(n^{2}-1\right) / 3 .
$$

Из (55) и (53) следует (51).

Пусть $x \in\left[x_{0}, \bar{x}\right]$, где $\bar{x} \in \partial K$ и $x \neq \bar{x}$. Пусть $P$ - параллелограмм с вершиной в точке $\bar{x}$, центром в точке $x_{0}$ и сторонами $u_{1}=y_{1}-\bar{x}, u_{2}=y_{2}-\bar{x}$. Точке $x \in P$ в представлении

$$
x=x_{0}-\left(t_{1} u_{1}+t_{2} u_{2}\right) / 2
$$

соответствуют $t_{1}(x)=t_{2}(x)$. Поэтому из обобшенного неравенства Бернштейна (32) вытекает неравенство

$$
\left|\left\langle\nabla P_{n}(x), y_{1}-y_{2}\right\rangle\right| / 2 \leqslant \sqrt{2}\left\|\nabla U_{n}\left(t_{1}(x), t_{1}(x)\right)\right\|_{\ell_{2}^{(2)}} \leqslant \sqrt{2} n /\left(1-t_{1}^{2}(x)\right)^{1 / 2} .
$$

Так как $\rho\left(x, x_{0}\right)=\left|t_{1}(x)\right|$, то из (56) следует (50).

СлЕДСТвИЕ 7. Если $X$ - гильбертово пространство, то в условиях теоремы 5 оценка (51) есть оценка $\left\|\nabla^{2} P_{n}\right\|_{K}[13$, с. 75$]$ и имеют место неравенства

$$
\begin{aligned}
\left\|\nabla^{k} P_{n}\right\|_{K} \leqslant & \left(4 /\left(3 r^{2}(K)\right)\right)^{p} \prod_{i=0}^{p-1}(n-2 i)(n-2 i-1 / 2) \\
& \times\left((n-2 i)^{2}-1\right)=\widetilde{M}_{2 p}, \quad k=2 p, \quad p \geqslant 1 ; \\
\left\|\nabla^{k} P_{n}\right\|_{K} \leqslant & \sqrt{2} \widetilde{M}_{2 p}(n-2 p)^{2} / r(K), \quad k=2 p+1 .
\end{aligned}
$$

Если $X$ - банахово пространство, то справедливо неравенство

$$
\left\|\nabla^{k} P_{n}\right\|_{K} \leqslant 2^{k / 2} \prod_{i=0}^{k-1}(n-i)^{2} / r^{k}(K) .
$$

Теорема 6. Пусть на $\mathbb{R}^{2}$ задана норма $\|\cdot\|$ и $K$ - параллелограмм, принадлежсащий $\mathbb{R}^{2}$. Пусть $P_{n}(x) \in \mathfrak{P}_{n}\left(\mathbb{R}^{2}, \mathbb{R}\right)$ u $\left\|P_{n}\right\|_{K} \leqslant 1$. Тогда имеет место неравенство

$$
\left\|\nabla^{2} P_{n}\right\|_{K} \leqslant C_{2} / r^{2}(K) .
$$

ДокАЗАТЕЛЬСтво. Пусть $d_{1}$ и $d_{2}$ - векторы полудиагоналей параллелограмма $K$. Точно так же, как в теореме 5 , доказывается, что имеют место неравенства

$$
\left|\left\langle\nabla^{2} P_{n}(x) d_{i}, d_{j}\right\rangle\right| \leqslant C_{2}, \quad x \in K, \quad i, j=1,2 .
$$

Пусть $h_{1}$ и $h_{2}$ - векторы, имеюшие начало в центре $K$ и принадлежашие $K$. Тогда $h_{i}=\alpha_{1}^{i} d_{1}+\alpha_{2}^{i} d_{2}$, где $\left|\alpha_{1}^{i}\right|+\left|\alpha_{2}^{i}\right| \leqslant 1$, и справедливо неравенство

$$
\left|\left\langle\nabla^{2} P_{n}(x) h_{1}, h_{2}\right\rangle\right| \leqslant\left(\left|\alpha_{1}^{1}\right|+\left|\alpha_{2}^{1}\right|\right)\left(\left|\alpha_{1}^{2}\right|+\left|\alpha_{2}^{2}\right|\right) C_{2}, \quad x \in K .
$$

Из (59) вытекает (57). Теорема доказана. 


\section{Список литературы}

1. Марков А.А. Об одном вопросе Д.И. Менделеева // Избранные труды. М.-Л.: ГИТТЛ, 1948.

2. Марков B. A. О функциях, наименее уклоняющихся от нуля в данном промежутке. СПб., 1892.

3. Бернштейн С. Н. Собрание сочинений. Т. 1. М.: Изд. АН СССР, 1952.

4. Бернштейн C. Н. Собрание сочинений. Т. 2. М.: Изд. АН СССР, 1954.

5. Kellogg O. D. On bounded polynomials on several variables // Math. Zeit. 1927. B. 27. № 1. S. 55-66.

6. Don R. Wilhelmsen A. Markov Inequality in Several Dimensions // J. of Approx. Theory. 1974. V. 11. № 3.

7. Наджммиддинов Д., Субботин Ю. Н. Неравенство Маркова на треугольниках // Матем. заметки. 1989. Т. 46. № 2. С. 76-82.

8. Андрианов A. В. Аналоги неравенств А. Маркова и С. Бернштейна для многочленов в банаховых пространствах // Матем. заметки. 1992. Т. 52. № 5. С. 13-21.

9. Кларк $\Phi$. Оптимизация и негладкий анализ. М.: Наука, 1988.

10. Красносельский $M$. А. Оператор сдвига по траекториям дифференциальных уравнений. М.: Наука, 1966.

11. Ганзбург М. И. Теоремы Джексона и Бернштейна в $\mathbb{R}^{m} / /$ УМН. 1979. Т. 34. № 1 . C. $225-226$.

12. Schaffer A.S., Duffin R.J. On some inequalities of S. Bernstein and W. Markoff for derivatives of polynomials // Bull. Am. Math. Soc. 1938. V. 44. P. 289-297.

13. Ахиезер Н. И., Глазман И. М. Теория линейных операторов в гильбертовом пространстве. М.: Наука, 1966.

Поступило в редакцию 30.VI.1995 